\title{
Weight Influence of Logarithmic and Exponential Functions on the Selection of Wireless Networks Using Multi-Criteria Decision-Making Methods
}

\author{
https://doi.org/10.3991/ijim.v16i01.24545 \\ Abdulhakim Zentani ${ }^{(凶)}$, Nadiatulhuda Zulkifli, Arnidza Ramli \\ Faulty of Engineering, Universiti Teknologi Malaysia, Johor Bahru, Malaysia \\ mehemed1975@graduate.utm.my
}

\begin{abstract}
This research aims to study the influence of logarithmic and exponential functions on the multi-criteria decision-making algorithm that changes the linear to the nonlinear method. It is carried out to better understand the multi-criteria decision-making (TOPSIS) algorithm whereby these functions may influence the criteria weights during the selection of the best network. The experiment is applied under different network types to evaluate the most optimum network that leads to better throughput, low latency, minimum BER, and low price per MB. The algorithms are assessed in MATLAB simulation environments. In addition, the adoption of the Wi-Fi networks standard is determined by factors such as bandwidth, signal to noise ratio and the channel modulation technique during the decision-making process. The simulation results showed that the exponential function had produced approximately similar results to that of linear TOPSIS algorithm because both keep the weights to demonstrate positive values. However, logarithmic TOPSIS produced different results and a worstcase scenario, as the weights have negative values which lead to a phase shift of $180^{\circ}$ during the decision process. Thus, linear TOPSIS was found to have the best results while logarithmic TOPSIS had the worst outcome.
\end{abstract}

Keywords—-multi criteria decision making, AHP, TOPSIS

\section{Introduction}

In most nations, consumer interest in mobile services is increasing because of the need for data access anywhere at all times. Moreover, the rise in communication infrastructures offers connectivity through wired and unwired technologies [1]. As such, network providers face issues in supporting users or enhancing the infrastructure. Different wireless network technologies are being implemented into the network to provide a smooth integration, and interoperability [2]. The aim of this paper is to pick the optimum candidate network in a distinct wireless environment. The study is done with three unique algorithms that factors in throughput, latency, BER and cost per $\mathrm{Mb}$ [3]. These factors affect the various networks during the selection phase, the multi-criteria decision-making (MCDM) algorithm exponential and logarithm functions, changing the linear to the nonlinear method. The paper starts with the theoretical background presented in Section 2. The simulation and setup are introduced in Section 3, and the results and discussion are described in Section 4. Finally, Section 5 concludes our work. 


\section{Theoretical background}

The MCDM techniques are employed for their decision-making capabilities used to choose the network nearest to the optimum standard and not the poorest one [4]. Numerous studies were done in the selection phase with a standard MCDM method called the Technique for Order Preference by Similarity to Ideal Solution (TOPSIS) technique to successfully deal with the handover decision-making process. Works in [5] [6] suggested a TOPSIS technique that factors in the price, total bandwidth, network usage, lag and interruptions. A separate study in [7] [8] proposed the formation and arrangement of the decision matrix using different parameters. According to [9] [10], the TOPSIS technique lowers limited connectivity in mixed networks lowering the handovers and packet loss while increasing the average user throughput [11]. However, the predetermined value utilized for weighing the handover metrics may have minor flaws, because of the diversity in signal power resulting from user movement in dynamic situations [12].

\subsection{Network performance parameters}

The criteria used in the selection algorithm are assessed by the TOPSIS algorithm, include throughput, latency, bit error rate (BER) and price per $\mathrm{Mb}$. We will explain the Signal-to-Noise Ratio (SNR) and bandwidth (B) that effects each criterion used in our research [13]. The noise power is calculated using the transmission signal bandwidth and the $n(t)$ spectral characteristics, where $n(t)$ is a white gaussian random noise with a zero mean and a power density of $\mathrm{N}_{0} / 2$. The noise power in bandwidth $2 \mathrm{~B}$ is defined by equation (1). Where, $\mathrm{N}_{0}$ is the noise power [14]. The SNR of the received signal can be given by equation (2), and $\mathrm{P}_{\mathrm{r}}$ is received power. SNR is usually defined as a function of the signal energy per bit $E_{b}$ or per symbol Es as in equation (3), $T_{s}$ is the symbol time while $\mathrm{T}_{\mathrm{b}}$ is time of the bit.

$$
\begin{gathered}
\mathrm{N}=\frac{\mathrm{N}_{0} \times 2 \mathrm{~B}}{2}=\mathrm{N}_{0} \mathrm{~B} \\
\mathrm{SNR}=\frac{\mathrm{P}_{\mathrm{r}}}{\left(\mathrm{N}_{0} \mathrm{~B}\right)} \\
\mathrm{SNR}=\frac{\mathrm{P}_{\mathrm{r}}}{\left(\mathrm{N}_{0} \mathrm{~B}\right)}=\frac{\mathrm{E}_{\mathrm{S}}}{\left(\mathrm{N}_{0} \mathrm{BT}_{\mathrm{S}}\right)}=\frac{\mathrm{E}_{\mathrm{b}}}{\left(\mathrm{N}_{0} \mathrm{BT}_{\mathrm{b}}\right)}
\end{gathered}
$$

For average power S constraints, Shannon channel capacity with a channel-side information (CSI) receiver can be gained as described in the equation $=\mathrm{S} /\left(N_{0} \mathrm{~B}\right)$. The efficiency of Shannon is equivalent to the power of Shannon of AWGN noise with throughput $=\mathrm{B} \log 2(1+\mathrm{SNR})$ and is distributed over SNR. Therefore, the capacity of Shannon is also known as the capacity of Ergodic [15] [16]. Considering a discrete-time AWGN channel having the relationship between bandwidth and power, the signal-to-noise ratio is constant and defined by (4) [17].

$$
\mathrm{SNR}=\mathrm{S} /\left(\mathrm{N}_{0} \mathrm{~B}\right)
$$


Throughput. Shannon's equation that $\mathrm{C}=\mathrm{B} \log 2(1+\mathrm{SNR})$ illustrates the capacity of interruption is applied to slowly varying channels where the SNR can be considered fixed over a large number of transmissions. After the burst, it changes value according to the fading criteria. In this model, if the communication channel has accepted a given SNR during a transmission, data can be sent through the communication channel at throughput as in equation (5) [18] [19]. The probability of outage declared by the transmitter is then by equation (6). The rate of the correctly received bits out of various transmission bursts can be given by equation (7) [20].

$$
\begin{gathered}
\mathrm{B} \log 2(1+\mathrm{SNR}) \\
\mathrm{P}_{\text {out }}=\mathrm{P}\left(\mathrm{SNR}<\mathrm{SNR}_{\min }\right) \\
\mathrm{C}_{\mathrm{o}}=\left(1-\mathrm{P}_{\text {out }}\right) \mathrm{B} \log _{2}\left(1+\mathrm{SNR}_{\min }\right)
\end{gathered}
$$

Latency. A QoS measure is packet latency from source to destination, such as video conferencing, gaming and Voice over Internet Protocol (VoIP) [21]. This parameter allows us to study packet queueing delays [22] that consists of four elements. First, the transmission delay (packet length/ throughput) is a function of packet length and network bandwidth (bps) [23]. Second, the delay of radio propagation is calculated by (distance/transmission speed) [24]. Third, the delay of queueing is defined by equation (8). Where $\mathrm{N}$ is the number of packets and $\mathrm{L}$ denotes the packet size.

$$
\text { Average queuing delay }=\left(\frac{\frac{(\mathrm{N}-1)}{\mathrm{L}}}{2 * \mathrm{C}}\right)
$$

Fourth, the processing delay in high-speed routers, in the range of microseconds or less, making them insignificant. Therefore, the total delay relationship can be expressed mathematically as $\mathrm{D}=$ Transmission delay + Radio propagation delay + Average queuing delay [25].

Bit error rate. In communication systems, BER is a significant metric for assessing system performance. For example, in simple systems where the channel is simplified by the additive white gaussian noise (AWGN), the BER is found quickly [26]. The required power to keep a probability of error $\left(\mathrm{P}_{\mathrm{b}}\right)$ small in fading channels is greater than in AWGN channels. The BER expression for M-QAM as in equation (9), where M is order of M-array and the $\mathrm{SNR}=\mathrm{Eb} / \mathrm{No}$ [27].

$$
P_{b}=\frac{\sqrt{M}-1}{\sqrt{M} \log _{2} \sqrt{M}} \operatorname{erfc}\left(\sqrt{\frac{3\left(\log _{2} M\right) E_{b}}{2(M-1) N_{0}}}\right)
$$

Price per Mb. The price per $1 \mathrm{Mb}$ is equal to some values (\$), so the price increases when the throughput increases [28]. Therefore, it can get the price per Mb by equation (10).

$$
\text { Price per } \mathrm{Mb}=\frac{\text { price }}{1 \times 10^{6}} \times \text { Throughput. }
$$


Packet length and packets size. Packet length is measured between network server and Internet connection. The central limit theorem may deduce that the aggregate traffic has a Gaussian distribution due to several devices generate the packets. Therefore, when data traffic flows through the aggregation point, it suffers a non-linear transformation, and the network servers group the packets (bytes) according to the adopted protocol (IP, ICMP, TCP or UDP), producing a non-uniform distribution for the packet size [29] [30].

The non-linear transformation of the Beta distribution gives the bimodal distribution [31]. For the ethernet network standard, the Maximum Traffic Unit (MTU) is 1500 bytes. The maximum packet length that can be sent through a network interface is 1492 bytes because eight bytes are used in the logical link control (LLC) header [32]. It can be good to only send one larger packet instead of multiple small ones [33]. The negative impact is that one big packet will take up space in the buffers, creating more packet loss if the buffer is not large enough to handle the incoming packets. The theoretical max size for TCP and UDP is around $64 \mathrm{kB}$ (TCP 65535 bytes and UDP 65507 bytes) [34] because of the MTU (Maximum Transmission Unit), which is the largest size of data that can be sent at a time. If data with a size larger than the MTU is passed from the transport layer to the network layer, it will be fragmented into smaller packages provided with the IP header with the final destination address. The MTU size used for the simulation was one with packet size of 1000 bytes payload [35]. For all scenarios, real-time video is expected to be conveyed using RTP/UDP/IP packets.

The number of packets. The transmission generates packet losses during the transmission of digital video signals through a packet switching system. Therefore, the time required for the packet in transmission medium is $\mathrm{Np}=$ Number of packets, $\mathrm{d}=$ delay, then the size of the packet is $\mathrm{Sp}=\mathrm{d} / \mathrm{Np}$. Figure 1 shows The header of the video signal transmitter, according to [36] [37].
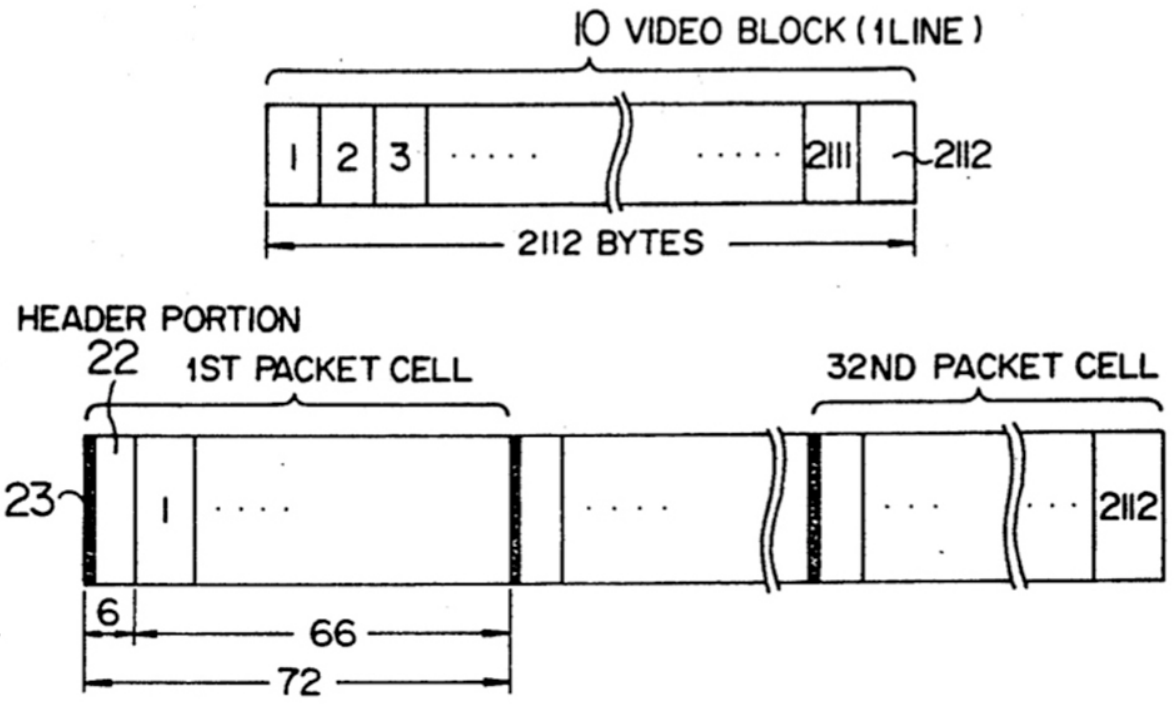

Fig. 1. The header of video signal transmitter 


\subsection{Analytic hierarchy process (AHP)}

This method assesses the weight of different criteria of a candidate network [38] [39]. A pairwise comparison approach is used and entered into a matrix and used to determine a vector of priority weights [40]. Therefore, to calculate the pairwise defined by (11), where, $x_{i i}=1$, and $x_{i j}$ elements are obtained from Table 1, (Saaty table) for pairwise comparison technique.

$$
\left[\begin{array}{l}
x_{11} x_{12} \ldots \ldots \ldots x_{1 n} \\
x_{21} x_{22} \ldots \ldots \ldots x_{2 n} \\
x_{n 1} x_{n 2} \ldots \ldots \ldots x_{n n}
\end{array}\right]
$$

Note that: $W_{i}$ is a weight for attribute $i, i=1$ to $n$, where $n=$ number of attributes and $\left(x_{i j}=x_{i / x_{j}}\right)$ the result of a pairwise comparison between attribute $i$ as compared to attribute $j$ as shown in Table 1. Therefore, the normalized as defined by (12), where, $r_{i j}=x_{i j} / \sum_{i=1}^{n} x_{i j}$, the weight can be calculated by equation (13), $W_{i}$ the assigned weight for every parameter and that $\sum_{j=1}^{n} W_{i}=1[41]$.

$$
\begin{gathered}
{\left[\begin{array}{c}
r_{11} r_{12} \ldots \ldots \ldots r_{1 n} \\
r_{21} r_{22} \ldots \ldots \ldots r_{2 n} \\
r_{n 1} r_{n 2} \ldots \ldots \ldots r_{n n}
\end{array}\right]} \\
W_{i}=\frac{\sum_{j=1}^{n} r_{i j}}{n}
\end{gathered}
$$

Table 1. Saaty scales for pairwise comparison technique

\begin{tabular}{|c|l|}
\hline Saaty Scales & \multicolumn{1}{|c|}{ The Relative Importance of the Two Sub-Elements } \\
\hline 1 & Equally important. \\
\hline 3 & Moderately important \\
\hline 5 & Strongly important. \\
\hline 7 & Very strong important. \\
\hline 9 & Extremely important. \\
\hline $2,4,6,8$ & Intermediate value. \\
\hline
\end{tabular}

\subsection{TOPSIS algorithm}

The linear-TOPSIS algorithm is used to find the best solution for the system under different conditions for each metric [42] and [43]. The steps of the TOPSIS algorithm are:

- Construct the decision matrix (DM) as specified by (14), where network1 and network 2 are two feasible options from which the decision-makers must choose, and $\mathrm{C}_{1}, \mathrm{C}_{2}, \mathrm{C}_{3}$ and $\mathrm{C}_{4} \cdot \mathrm{x}_{\mathrm{ij}}$ are the ratings of an alternative based on criteria [44]. 


$$
\mathrm{DM}=\left[\begin{array}{cccc}
\mathrm{C}_{1} & \mathrm{C}_{2} & \mathrm{C}_{3} & \mathrm{C}_{4} \\
\mathrm{x}_{11} & \mathrm{x}_{12} & \mathrm{x}_{13} & \mathrm{x}_{14} \\
\mathrm{x}_{21} & \mathrm{x}_{22} & \mathrm{x}_{23} & \mathrm{x}_{24}
\end{array}\right]
$$

- Construction of the Normalized Decision Matrix, as shown in the equation (15), where $r_{i j}$ the normalization value, $i=1,2, \ldots \mathrm{m}$, and $\mathrm{j}=1,2, \ldots \mathrm{n}$ to convert the dimensional attributes into non-dimensional, ones to compare between different attributes [45].

- Construct the Weighted Normalized Decision Matrix as in equations (16) and the effect of each function on the weight, where $\mathrm{w}_{\mathrm{i}}$ is the weight of criterion $\mathrm{r}_{\mathrm{ij}}$

$$
r_{i j}=\frac{x_{i j}}{\sqrt{\sum_{i=1}^{m} x_{i j}^{2}}}=\left[\begin{array}{llll}
r_{11} & r_{12} & r_{13} & r_{14} \\
r_{21} & r_{22} & r_{23} & r_{24}
\end{array}\right]
$$

- First construct linear-TOPSIS.

$$
\mathrm{v}_{\mathrm{ij}}=\left[\begin{array}{llll}
\mathrm{r}_{11} \times \mathrm{w}_{1} & \mathrm{r}_{12} \times \mathrm{w}_{2} & \mathrm{r}_{13} \times \mathrm{w}_{3} & \mathrm{r}_{14} \times \mathrm{w}_{4} \\
\mathrm{r}_{21} \times \mathrm{w}_{1} & \mathrm{r}_{22} \times \mathrm{w}_{2} & \mathrm{r}_{23} \times \mathrm{w}_{3} & \mathrm{r}_{24} \times \mathrm{w}_{4}
\end{array}\right]
$$

- Second construct Exponential-TOPSIS by defined (17).

$$
\mathrm{v}_{\mathrm{ij}}=\left[\begin{array}{llll}
\mathrm{r}_{11} \times \text { Exp. } \mathrm{w}_{1} & \mathrm{r}_{12} \times \operatorname{Exp} \times \mathrm{w}_{2} & \mathrm{r}_{13} \times \text { Exp. }_{3} & \mathrm{r}_{14} \times \operatorname{Exp} \times \mathrm{w}_{4} \\
\mathrm{r}_{21} \times \text { Exp. } \mathrm{w}_{1} & \mathrm{r}_{22} \times \operatorname{Exp} \times \mathrm{w}_{2} & \mathrm{r}_{23} \times \text { Exp. } \mathrm{w}_{3} & \mathrm{r}_{24} \times \operatorname{Exp} \times \mathrm{w}_{4}
\end{array}\right]
$$

- Third construct Logarithmic-TOPSIS by defined (18).

$$
\mathrm{v}_{\mathrm{ij}}=\left[\begin{array}{llll}
\mathrm{r}_{11} \times \log \times \mathrm{w}_{1} & \mathrm{r}_{12} \times \log \times \mathrm{w}_{2} & \mathrm{r}_{13} \times \log \times \mathrm{w}_{3} & \mathrm{r}_{14} \times \log \times \mathrm{w}_{4} \\
\mathrm{r}_{21} \times \log \times \mathrm{w}_{1} & \mathrm{r}_{22} \times \log \times \mathrm{w}_{2} & \mathrm{r}_{23} \times \log \times \mathrm{w}_{3} & \mathrm{r}_{24} \times \log \times \mathrm{w}_{4}
\end{array}\right]
$$

- Determine Ideal and Negative-Ideal Solutions.

$\mathrm{A}^{+}=\left\{\mathrm{v}_{1}^{+}, \mathrm{v}_{2}^{+}, \ldots \ldots\right\}, \mathrm{v}_{\mathrm{j}}^{+}=\max _{\mathrm{i}}\left(\mathrm{v}_{\mathrm{ij}}\right)$, associated with benefit or best criteria.

$\mathrm{A}^{-}=\left\{\mathrm{v}_{1}^{-}, \mathrm{v}_{2}^{-}, \ldots \ldots\right\}, \mathrm{v}_{\mathrm{j}}^{-}=\min _{\mathrm{i}}\left(\mathrm{v}_{\mathrm{ij}}\right)$, associated with cost or worse criteria.

- Calculate the solution measure as shown in equations (19) and (20):

$$
\begin{aligned}
& \mathrm{S}_{\mathrm{i}}^{+}=\sqrt{\sum_{\mathrm{j}=1}^{\mathrm{m}}\left|\mathrm{v}_{\mathrm{i}}^{+}-\mathrm{v}_{\mathrm{ij}}\right|}, \mathrm{i}=1,2, \ldots \mathrm{m} \text { (positive-ideal-solution) } \\
& \mathrm{S}_{\mathrm{i}}^{-}=\sqrt{\sum_{\mathrm{j}=1}^{\mathrm{m}}\left|\mathrm{v}_{\mathrm{i}}^{-}-\mathrm{v}_{\mathrm{ij}}\right|}, \mathrm{i}=1,2, \ldots \mathrm{m} \text { (positive-ideal-solution) }
\end{aligned}
$$

- Calculate the proportional proximity to the Ideal-solution $\mathrm{C}_{\mathrm{i}}$, like in equation (21). Where, $0<\mathrm{C}_{\mathrm{i}}<1,\{1,2, \ldots \mathrm{m}\}, \mathrm{C}_{\mathrm{i}}=1$ if $\mathrm{S}_{\mathrm{i}}=\mathrm{S}^{+}$also $\mathrm{C}_{\mathrm{i}}=0$ if $\mathrm{S}_{\mathrm{i}}=\mathrm{S}$

$$
\mathrm{C}_{\mathrm{i}}=\frac{\mathrm{S}_{\mathrm{i}}^{-}}{\mathrm{S}_{\mathrm{i}}^{+}+\mathrm{S}_{\mathrm{i}}^{-}}
$$




\section{Simulation and setup}

The study demonstrates how weights and attributes influence the scoring values and efficiency functions of linear-TOPSIS algorithms, logarithmic and exponential algorithms. Varying parameters were tested and are illustrated in this section through MATLAB simulation. With regards to Table 2, we use three different Wi-Fi networks as the topology based on (IEEE) 802.11 standards. In addition, the SNR values, bandwidth, and M-QAM modulation techniques for all the Wi-Fi networks vary, as shown in Figure 2.

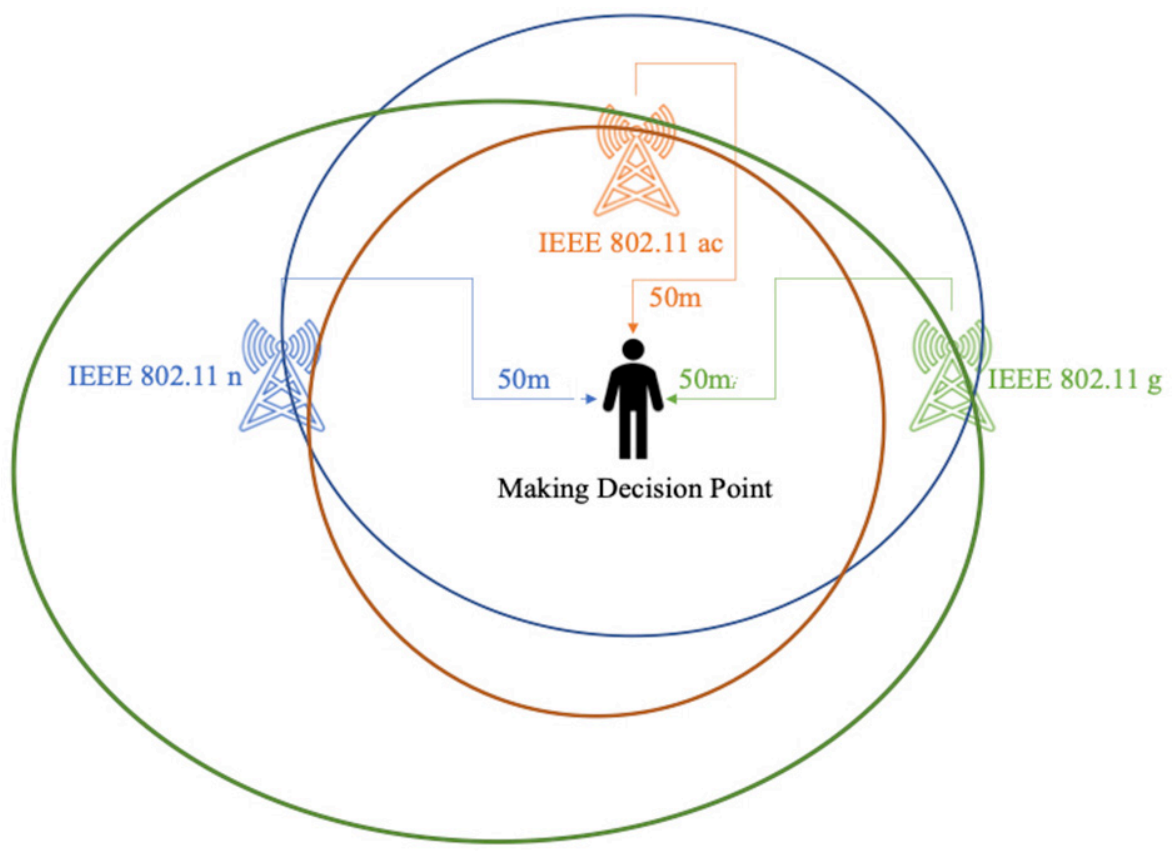

Fig. 2. Network topology simulation

The price per MB is equivalent to $\$ 0.05$, the length per packet is 1492 , and the number of packets is 260 as noted by [37]. The values of a packet per size are equal to 1000 as noted in [35], and the distance is $50 \mathrm{~m}$. The relative significance of various attributes is examined in relation to the weights, through the use of a of pair-wise comparison based on the scale of relative importance (AHP method) as explained in Section 2.2. We selected four criteria with each having values out of 1 . From Saaty scales for pairwise comparison technique Table 1 in Section 2.2, we rank throughput as extremely important $(0.4375)$, latency as very important $(0.3125)$, the price per Mb as moderately important $(0.1875)$, and BER as equally important $(0.0625)$ for linear-TOPSIS simulation to be suitable for video streaming services. To determine the performance of the selection process, we utilized the following metrics throughput, latency, packet loss (BER) and price per $\mathrm{Mb}$, to attain reliable results in Table 2. 


\section{$4 \quad$ The results and discussion}

In the Linear-TOPSIS, Figure 3a illustrates that the algorithm picks the ideal candidate network based on the high throughput value and latency even with the high price of data rate flow as in Table 2. The method then selects a substitute most similar to the optimum solution. TOPSIS consideration of each attribute either takes a gradual increasing or decreasing network. The graph in Figure $3 \mathrm{c}$ illustrates the results of selection networks with exponential-TOPSIS algorithm, producing the same results when compared with the linear-TOPSIS. Figure $3 \mathrm{~b}$ presents the results of three networks with logarithmic-TOPSIS algorithm. The change of weight resulted in negative values before normalizing. However, after normalizing, the algorithm picks the poorest network as the best candidate network. Thereby, we found that the linear and exponential TOPSIS algorithms have the same result and will constantly pick the ideal candidate network compared to logarithmic TOPSIS algorithm which instead, chooses the poorest network.

Table 2. The basic parameters for TOPSIS algorithm

\begin{tabular}{|c|c|c|c|}
\hline Network Parameters & IEEE $802.11 \mathrm{~g}$ & IEEE $802.11 \mathrm{n}$ & IEEE 802.11 ac \\
\hline SNR & 40 & 30 & 20 \\
\hline BW & 20 & 40 & 80 \\
\hline Channel/modulation & 256-QAM & 64-QAM & 16-QAM \\
\hline Throughput (MB) & 259 & 385 & 506 \\
\hline Latency $(\mathrm{ms})$ & 0.0747 & 0.0374 & 0.0188 \\
\hline Price per MB (\$) & 12.95 & 19.27 & 25.31 \\
\hline $\operatorname{BER}\left(10^{-3}\right)$ & 0.0015 & 0.0022 & 0.0006 \\
\hline Distance & \multicolumn{3}{|c|}{$50 \mathrm{~mm}$} \\
\hline Price per Mb [\$] & \multicolumn{3}{|c|}{$0.05 \$$} \\
\hline The number of packets & \multicolumn{3}{|c|}{260} \\
\hline Packet per size & \multicolumn{3}{|c|}{1000} \\
\hline Length of packet & \multicolumn{3}{|c|}{1492} \\
\hline
\end{tabular}



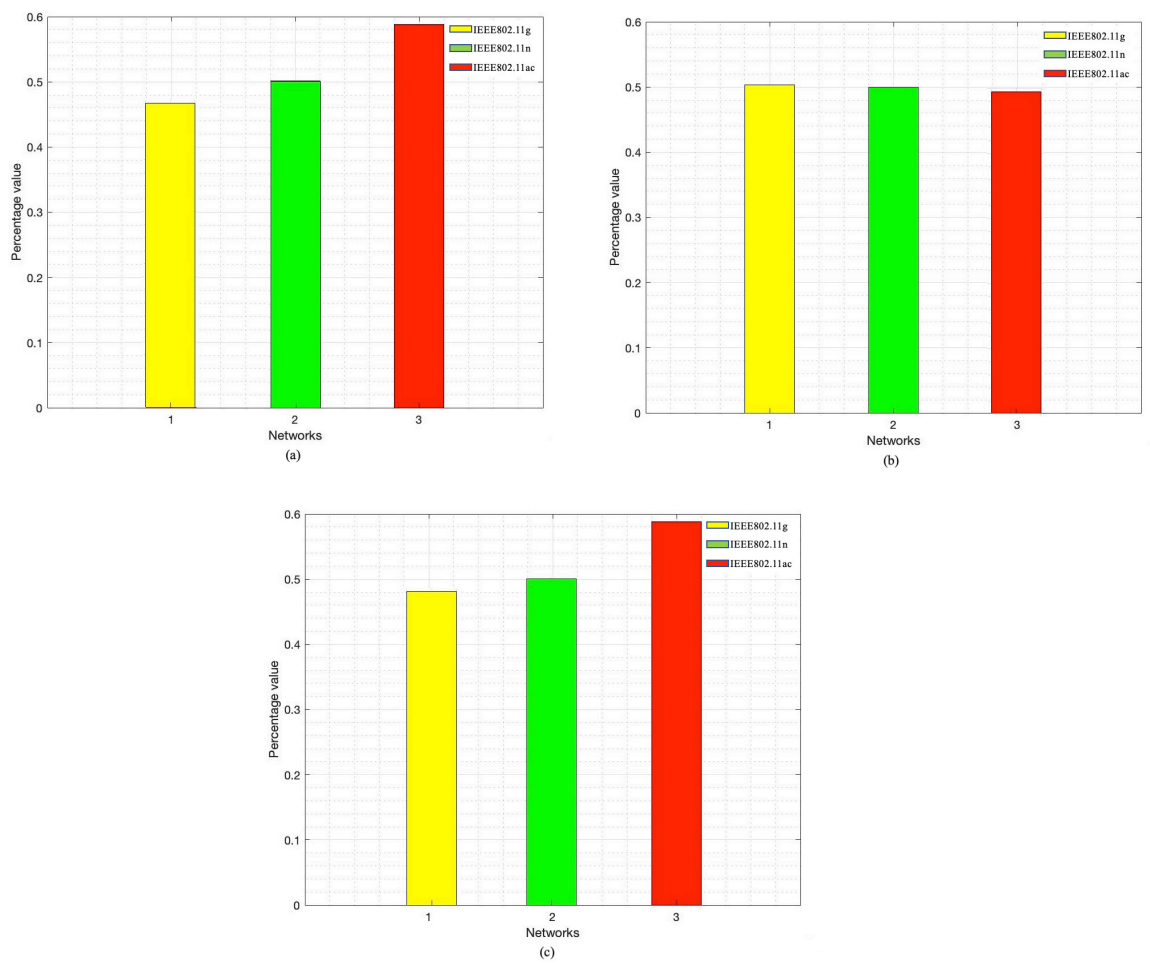

Fig. 3. The weightage versus network types for (a) linear-TOPSIS, (b) logarithmic-TOPSIS and (c) exponential-TOPSIS

\section{Conclusions}

In wireless networks, uninterrupted connection is a vital factor, with the need to prevent connection drop experienced by users in dynamic situations. This paper has focused on implementing the TOPSIS algorithm to select the best network to be used by a network client. The TOPSIS algorithm is implemented in the MATLAB environment and is examined under various parameter values. Different functions (logarithmic and exponential) affect the weights given for each attribute in the TOPSIS algorithm. The values of each weight are varied to show the effect of each function on the decision. We found out that the various functions have made other choices for each network under the same parameters. Besides, the simulation results showed that the use of functions affects the network selection predominantly. This effect demonstrated through the simulation of various weight parameters. Therefore, Linear-TOPSIS has given the best results, while logarithmic TOPSIS has produced the worst consequences. This research opens the doors widely to investigating modern intelligent algorithms like neural networks, fuzzy logic and other MCDM techniques like Gray Relational Analysis (GRE), 
Simple Adaptive Weighting (SAW) and Multiplicative Exponent Weighting (MEW)) to study the effects of these functions by using services such as video streaming, VoIP, data browsing in dynamic situations.

\section{Acknowledgement}

We thank the Ministry of High Education Malaysia (MOHE) and Research Management Center of Universiti Teknologi Malaysia, for financial support under Transdisciplinary Research Grant (Vot No 05G60). We also thank the sponsor Ministry of Higher Education and Scientific Research Libya for the scholarship provided to the first author.

\section{$7 \quad$ References}

[1] G. Lampropoulos, C. Skianis, and P. Neves, "Optimized fusion of heterogeneous wireless networks based on media-independent handover operations [accepted from open call]," IEEE Wirel. Commun., vol. 17, no. 4, pp. 78-87, 2010. https://doi.org/10.1109/ MWC.2010.5547925

[2] T. S. Rappaport, "Wireless communications_principles and practice (The Book End)," Microw. J., vol. 45, no. 12, pp. 128-129, 2002.

[3] J. M. Marquez-Barja et al., "Breaking the vehicular wireless communications barriers: Vertical handover techniques for heterogeneous networks," IEEE Trans. Veh. Technol., vol. 64, no. 12, pp. 5878-5890, 2015. https://doi.org/10.1109/TVT.2014.2386911

[4] C. Yeh, "A problem-based selection of multi-attribute decision-making methods," Int. Trans. Oper. Res., vol. 9, no. 2, pp. 169-181, 2002. https://doi.org/10.1111/1475-3995.00348

[5] L. Wang and D. Binet, "MADM-based network selection in heterogeneous wireless networks: A simulation study," in 2009 1st International Conference on Wireless Communication, Vehicular Technology, Information Theory and Aerospace \& Electronic Systems Technology, 2009, pp. 559-564. https://doi.org/10.1109/WIRELESSVITAE.2009.5172507

[6] L. Wang and G.-S. Kuo, "Mathematical modeling for network selection in heterogeneous wireless networks-A tutorial," IEEE Commun. Surv. Tutorials, vol. 15, no. 1, pp. 271-292, 2012. https://doi.org/10.1109/SURV.2012.010912.00044

[7] B. Bakmaz, Z. Bojkovic, and M. Bakmaz, "Network selection algorithm for heterogeneous wireless environment," in 2007 IEEE 18th International Symposium on Personal, Indoor and Mobile Radio Communications, 2007, pp. 1-4. https://doi.org/10.1109/ PIMRC.2007.4394315

[8] K. S. S. Anupama, S. S. Gowri, and B. P. Rao, "A comparative study of outranking MADM algorithms in network selection," in 2018 Second international conference on computing methodologies and communication (ICCMC), 2018, pp. 904-907. https://doi.org/10.1109/ ICCMC.2018.8487931

[9] X. Li and X. Chen, "Multi-criteria group decision making based on trapezoidal intuitionistic fuzzy information," Appl. Soft Comput., vol. 30, pp. 454-461, 2015. https://doi. org/10.1016/j.asoc.2015.01.054

[10] M. Alhabo and L. Zhang, "Multi-criteria handover using modified weighted TOPSIS methods for heterogeneous networks," IEEE Access, vol. 6, pp. 40547-40558, 2018. https://doi. org/10.1109/ACCESS.2018.2846045 
[11] K. Q. M. Alborahy and S. M. Sharif, "Enhancing the handover performance in the mobile heterogeneous network," Univ. Khartoum Eng. J., vol. 9, no. 1, 2019. https://doi. org $/ 10.1155 / 2019 / 8587932$

[12] R. Tiwari and S. Deshmukh, "MVU estimate of user velocity via gamma distributed handover count in HetNets," IEEE Commun. Lett., vol. 23, no. 3, pp. 482-485, 2019. https://doi. org/10.1109/LCOMM.2019.2892962

[13] P. A. Anghel and M. Kaveh, "Exact symbol error probability of a cooperative network in a Rayleigh-fading environment," IEEE Trans. Wirel. Commun., vol. 3, no. 5, pp. 1416-1421, 2004. https://doi.org/10.1109/TWC.2004.833431

[14] J. Miranda et al., "Path loss exponent analysis in wireless sensor networks: Experimental evaluation," in 2013 11th IEEE international conference on industrial informatics (INDIN), 2013, pp. 54-58. https://doi.org/10.1109/INDIN.2013.6622857

[15] A. Maaref and S. Aissa, "Closed-form expressions for the outage and ergodic Shannon capacity of MIMO MRC systems," IEEE Trans. Commun., vol. 53, no. 7, pp. 1092-1095, 2005. https://doi.org/10.1109/TCOMM.2005.851564

[16] K. Balachandran, S. R. Kadaba, and S. Nanda, "Channel quality estimation and rate adaptation for cellular mobile radio," IEEE J. Sel. Areas Commun., vol. 17, no. 7, pp. 1244-1256, 1999. https://doi.org/10.1109/49.778183

[17] J. G. Andrews, F. Baccelli, and R. K. Ganti, "A tractable approach to coverage and rate in cellular networks," IEEE Trans. Commun., vol. 59, no. 11, pp. 3122-3134, 2011. https://doi. org/10.1109/TCOMM.2011.100411.100541

[18] H. Huang, C. B. Papadias, and S. Venkatesan, MIMO communication for cellular networks. Springer Science \& Business Media, 2011. https://doi.org/10.1007/978-0-387-77523-4

[19] O. Simeone et al., Cooperative wireless cellular systems: An information-theoretic view. Now, 2012. https://doi.org/10.1561/9781601985491

[20] A. El Gamal and Y.-H. Kim, Network information theory. Cambridge university press, 2011. https://doi.org/10.1017/CBO9781139030687

[21] J.-C. Bolot, "End-to-end packet delay and loss behavior in the Internet," in Conference proceedings on Communications architectures, protocols and applications, 1993, pp. 289-298. https://doi.org/10.1145/167954.166265

[22] C. J. Bovy, H. T. Mertodimedjo, G. Hooghiemstra, H. Uijterwaal, and P. Van Mieghem, "Analysis of end-to-end delay measurements in Internet," in Proc. of the Passive and Active Measurement Workshop-PAM, 2002, vol. 2002.

[23] I. Maslouhi, E. Miloud, K. Ghoumid, and K. Baibai, "Analysis of end-to-end packet delay for Internet of Things in wireless communications," Int. J. Adv. Comput. Sci. Appl, vol. 9, no. 9, pp. 338-343, 2018. https://doi.org/10.14569/IJACSA.2018.090944

[24] M. Kazantzidis and M. Gerla, "End-to-end versus explicit feedback measurement in 802.11 networks," in Proceedings ISCC 2002 Seventh International Symposium on Computers and Communications, 2002, pp. 429-434.

[25] Y. Chen, "Mathematical modelling of end-to-end packet delay in multi-hop wireless networks and their applications to qos provisioning." University College London (University of London), 2013.

[26] J. Van Wyk and L. Linde, "Bit error probability for a M-ary QAM OFDM-based system," in AFRICON 2007, 2007, pp. 1-5. https://doi.org/10.1109/AFRCON.2007.4401578

[27] D. Yoon, K. Cho, and J. Lee, "Bit error probability of M-ary quadrature amplitude modulation," in Vehicular Technology Conference Fall 2000. IEEE VTS Fall VTC2000. 52nd Vehicular Technology Conference (Cat. No. 00CH37152), 2000, vol. 5, pp. 2422-2427.

[28] S. Singh, M. S. Kumar, and H. S. Mruthyunjaya, "A new generalized closed form expression for average bit error probability over Rayleigh fading channel," arXiv Prepr. arXiv1210.0210, 2012. 
[29] J. Korhonen and Y. Wang, "Effect of packet size on loss rate and delay in wireless links," in IEEE Wireless Communications and Networking Conference, 2005, 2005, vol. 3, pp. 1608-1613.

[30] A. Lofgren, L. Lodesten, S. Sjoholm, and H. Hansson, "An analysis of FPGA-based UDP/IP stack parallelism for embedded Ethernet connectivity," in 2005 NORCHIP, 2005, pp. 94-97. https://doi.org/10.1109/NORCHP.2005.1596997

[31] L. Chaddad, A. Chehab, I. H. Elhajj, and A. Kayssi, "Mobile traffic anonymization through probabilistic distribution," Proc. 2019 22nd Conf. Innov. Clouds, Internet Networks Work. ICIN 2019, pp. 242-248, 2019. https://doi.org/10.1109/ICIN.2019.8685871

[32] J. Chi, S. Do, and S. Park, "Traffic flow-based roadside unit allocation strategy for VANET," 2016 Int. Conf. Big Data Smart Comput. BigComp 2016, pp. 245-250, 2016. https://doi. org/10.1109/BIGCOMP.2016.7425920

[33] B. Clément, "Linux Kernel packet transmission performance in high-speed networks," 2016.

[34] F. Ahmed, "Improving RSSI based distance estimation for wireless sensor networks," 2010.

[35] B. Preveze and A. Safak, "Throughput improvement of mobile multi-hop wireless networks," Int. J. Wirel. Mob. Networks, vol. 2, no. 3, 2010. https://doi.org/10.5121/ijwmn.2010.2309

[36] S. Pudlewski, N. Cen, Z. Guan, and T. Melodia, "Video transmission over lossy wireless networks: A cross-layer perspective," IEEE J. Sel. Top. Signal Process., vol. 9, no. 1, pp. 6-21, 2015. https://doi.org/10.1109/JSTSP.2014.2342202

[37] T. Kinoshita and Y. Eto, "Video signal transmitting method and equipment of the same." Google Patents, Oct. 27, 1992.

[38] S. Khanmohammadi and M. Rezaeiahari, "AHP based classification algorithm selection for clinical decision support system development," Procedia Comput. Sci., vol. 36, pp. 328-334, 2014. https://doi.org/10.1016/j.procs.2014.09.101

[40] T. L. Saaty, "The analytic hierarchy process Mcgraw Hill, New York," Agric. Econ. Rev., vol. 70, 1980. https://doi.org/10.21236/ADA214804

[41] R. K. Dewi, B. T. Hanggara, and A. Pinandito, "A comparison between AHP and Hybrid AHP for mobile based culinary recommendation system," Int. J. Interact. Mob. Technol., vol. 12, no. 1, pp. 133-140, 2018. https://doi.org/10.3991/ijim.v12i1.7561

[42] M. R. Asadabadi, E. Chang, and M. Saberi, "Are MCDM methods useful? A critical review of Analytic Hierarchy Process (AHP) and Analytic Network Process (ANP)," Cogent Eng., vol. 6, no. 1, 2019. https://doi.org/10.1080/23311916.2019.1623153

[43] G. R. Jahanshahloo, F. H. Lotfi, and M. Izadikhah, "An algorithmic method to extend TOPSIS for decision-making problems with interval data," Appl. Math. Comput., vol. 175, no. 2, pp. 1375-1384, 2006. https://doi.org/10.1016/j.amc.2005.08.048

[44] P. W. Bhutia and R. Phipon, "Application of AHP and TOPSIS method for supplier selection problem," IOSR J. Eng., vol. 2, no. 10, pp. 43-50, 2012. https://doi. org/10.9790/3021-021034350

[45] J. Liu and S. Li, "Research on the ranking of university education based on Grey-TOPSISDEA method," Int. J. Emerg. Technol. Learn., vol. 10, no. 8, pp. 51-54, 2015. https://doi. org/10.3991/ijet.v10i8.5279 


\section{Authors}

Abdulhakim Zentani, is a Ph.D. student in the School of Electrical Engineering Universiti Teknologi Malaysia. He received his higher diploma degree in Electrical and Electronic engineering from the High Advanced Center of Electronic-Tripoli-Libya in 2003 and his master's degree in Electrical and Electronic engineering in 2017 from Near East University Cyprus. His research interest is in the field of Fiber to Wireless network technologies. He is a researcher at Authority of Natural Science Research and Technology, Libya.

Nadiatulhuda Zulkifli is attached with Universiti Teknologi Malaysia UTM as an associate professor where her research interests include optical communication and networking. Prior to her Msc and $\mathrm{PhD}$ studies in University of Essex, UK from 2003 to 2009, she acquired BEng in Electrical-Telecommunication from Universiti Teknologi Malaysia. She was head of the Lightwave Communication Research Group from 2017 to 2019. Now, she is the coordinator for Master of Engineering in Electronics and Telecommunications.

Arnidza Ramli received both the Bachelor degree in Electrical Telecommunication and Master degree in Electrical from the Universiti Teknologi Malaysia (UTM) in 2007 and 2009 respectively. She completed her PhD study in Electrical Engineering from the same university in 2017. She is currently a Senior Lecturer at School of Electrical Engineering, Faculty of Engineering, UTM and serve in Communication Engineering Division. Her current research activity is mainly focused on Energy efficiency for integrated optical and wireless network. Other research interests include optical communication and green networking.

Article submitted 2021-06-04. Resubmitted 2021-07-08. Final acceptance 2021-07-14. Final version published as submitted by the authors. 